\title{
Dosimetric impact of volumetric modulated arc therapy for nasopharyngeal cancer treatment
}

\author{
Ciro Franzese $e^{1,2}$, Antonella Fogliata $\mathbb{Q}^{1}$, Mauro Loi ${ }^{1}$, Marco Badalamenti ${ }^{1}$, Davide Franceschini ${ }^{1}$, \\ Tiziana Comito ${ }^{1}$, Luca Cozzi ${ }^{1,2}$, Giacomo Reggiori ${ }^{1}$, Stefano Tomatis ${ }^{1}$, Marta Scorsetti ${ }^{1,2}$ \\ ${ }^{1}$ Humanitas Clinical and Research Hospital IRCCS, Radiotherapy Dept, Milan-Rozzano, Italy \\ ${ }^{2}$ Humanitas University, Biomedical Science Dept, Milan-Rozzano, Italy
}

\begin{abstract}
Background: The purpose of the study was to evaluate the toxicity and outcome of nasopharyngeal carcinoma patients treated using 3-dimensional conformal radiotherapy (3DCRT) or volumetric modulated arc therapy (VMAT) technique.

Materials and methods: 68 patients treated between 2006 and 2018 were retrospectively analysed. Since 2009 patients received 3DCRT with 50/70 Gy to the elective/boost volumes in 35 fractions; from then, VMAT with simultaneous integrated boost (SIB) with $54.45 / 69.96$ Gy in 33 , or $54 / 66$ Gy in 30 fractions. Induction chemotherapy was administered in $74 \%$ of the patients, concomitant cisplatinum in $87 \%$. Acute and late toxicity data, progression-free survival PSF and overall survival OS, and toxicity correlations with dose metrics were reported.

Results: With a median follow-up of 64 months, complete remission at the last evaluation was in $68 \%$ of the patients, while $28 \%$ and $9 \%$ had locoregional relapse and distant disease, respectively. The 5 - and 10 -year progression free survival (PFS) rates were $62.7 \pm 6.5 \%$ and $53.2 \pm 8.7 \%$, respectively. The 5 - and 10-year OS rates were $78.9 \pm 5.5 \%$ and $61.4 \pm 9.2 \%$, respectively At the multivariate Cox analysis TNM stage $(p=0.02)$ and concomitant chemotherapy $(p=0.01)$ resulted significant for PFS, concomitant chemotherapy $(p=0.04)$ for OS.

Improvements in acute toxicity were presented for VMAT patients due to its ability to spare OARs. Odds ratio (OR) for acute salivary toxicity, between VMAT and 3DCRT, was $4.67(p=0.02)$. Dosimetrically, salivary toxicity correlated with mean parotid dose $(p=0.05)$, dysphagia with laryngeal $(p=0.04)$ and mean oral cavity $(p=0.06)$ doses, when dose-volume histograms (DVHs) are corrected for fractionation.

Conclusion: This study is a proof of a significant benefit of the VMAT technique compared with 3DCRT in terms of side effects in nasopharynx patients, and adds dosimetric correlations.
\end{abstract}

Key words: nasopharyngeal cancer; VMAT; salivary toxicity; dysphagia

Rep Pract Oncol Radiother 2021;26(1):101-110

\section{Introduction}

Nasopharyngeal carcinoma (NPC) is a type of tumour characterized by different geographical incidence, with extremely high incidence in China and Southeast Asia [1]. The most common histological subtypes of NPC include keratinising squamous cell carcinoma (SCC), non-keratinizing differentiated carcinoma and non-keratinising undifferentiated carcinoma, classified by the World Health Organization (WHO) as subtypes I, II and III, respectively [2].

In non-endemic regions, including Europe, the majority of cases of NPC are EBV-negative kera-

Address for correspondence: Antonella Fogliata, Humanitas Clinical and Research Hospital IRCCS, Via Manzoni 56, 20089 Milan-Rozzano, Italy, fax: (+39) 02 82248509, tel: (+39) 338 7283355; e-mail: antonella.fogliata@humanitas.it

This article is available in open access under Creative Common Attribution-Non-Commercial-No Derivatives 4.0 International (CC BY-NC-ND 4.0) license, allowing to download articles and share them with others as long as they credit the authors and the publisher, but without permission to change them in any way or use them commercially 
tinising SCC or non-keratinising differentiated carcinoma, characterized by poorer survival and reduced rates of locoregional and distant disease control $[3,4]$.

Radiotherapy (RT), with or without concomitant chemotherapy, represents the standard of care for NPC. With the development of intensity-modulated radiation therapy (IMRT), a significant advantage in terms of healthy tissue sparing and improved local control was observed for NPC patients. With local control rates ranging between $88 \%$ and $97 \%$ [3, 5-7], and promising survival rates, IMRT is nowadays considered the standard RT technique for NPC. The benefit of the IMRT technique in NPC has been demonstrated also in terms of reduction of side effects, in particular salivary gland function and quality of life $[8,9]$. Kam et al. randomized 60 NPC patients to 2DRT versus IMRT with primary end-point to evaluate rates of xerostomia. At 1 year after treatment, patients treated with IMRT had a lower incidence of severe xerostomia than patients in the 2DRT arm (39.3\% vs. $82.1 \%$; $\mathrm{p}=0.001)$ [8]. More recently, to overcome limitations of the IMRT technique, arc therapy has been developed in the form of volumetric modulated arc therapy (VMAT). The concept of VMAT is the possibility to deliver radiation from a continuous rotation of the source and allows the patient to be continuously treated up to 360 degrees. The main advantage of VMAT compared to standard IMRT is the shorter treatment time. Additionally, relatively to certain IMRT deliveries (in particular the sliding window technique and those not based on direct aperture optimization), VMAT presents monitor unit and integral dose reductions, which could be of benefit for some side effects or second cancer induction risk [10-12].

However, despite the state of the art today is the VMAT (or IMRT) technique, according to an ESTRO survey on centres of 24 European countries $[13,14]$, in 2016 almost $30 \%$ of the interviewed institutions declared to treat the head and neck site with the 3-dimensional conformal radiotherapy (3DCRT) technique. This fact highlights that there is still the need to leverage, also in the developed countries, the treatment quality with the adoption of advanced technologies to reduce the side effects of radiotherapy.

Aim of the present study was to evaluate the pattern of toxicity and outcome of a cohort of NPC patients treated with radical RT, in the form of the 3DCRT or VMAT technique, with or without concomitant chemotherapy. Additionally, possible correlations between toxicity and dose metrics were assessed.

\section{Materials and methods}

\section{Patients selection}

From 2006 to 2018 a total of 68 patients affected by NPC received RT treatment in our institution. All patients were treated in agreement with the Helsinki declaration. Informed consent was obtained from all individual participants included in this study. A retrospective analysis of the treatment charts had been approved by our institutional ethical committee, and here reported. Patients were staged according to the American Joint Committee on Cancer (AJCC) staging system. Patient characteristics and treatment data are summarized in Table 1. Before treatment, patients had staging work-up through history, head and neck clinical examination, CT, MRI, and PET scans.

\section{Patient treatments}

The patients were all immobilised with individualised thermoplastic masks, in a supine position. A simulation CT with $3 \mathrm{~mm}$ slices and contrast-enhancing agent were acquired for all patients, co-registered with contrast-MRI and/or an 18FDG-PET to better define the targets. Based on the images, the gross tumour volume (GTV) was delineated. The high-risk clinical target volume (CTV) was defined as the GTV with an isotropic $1 \mathrm{~cm}$ margin, corrected for anatomical boundaries. The low-risk CTV included the elective nodes and was delineated according to internationally accepted guidelines $[15,16]$. A margin of $5 \mathrm{~mm}$ was added to both the CTVs to obtain the high- and low-risk planning target volumes (PTV). PTVs were limited to $5 \mathrm{~mm}$ within the patient outline for optimization purposes (while the evaluation was on the intact PTVs). The following organs at risk (OAR) were contoured: parotid glands, oral cavity, submandibular glands, larynx, thyroid, eyes, brainstem, and spinal cord.

The patients treated before 2009 received 3DCRT. In 2009, the VMAT technique (in the RapidArc form) became the standard in our institution. All treatments were planned on the Eclipse (Varian Medical Systems) treatment planning system. 
Table 1. Patients and disease characteristics

\begin{tabular}{|c|c|c|c|c|}
\hline \multirow{2}{*}{\multicolumn{2}{|c|}{ Number of patients }} & \multirow{2}{*}{$\begin{array}{l}\text { All } \\
68\end{array}$} & \multirow{2}{*}{$\begin{array}{c}\text { 3DCRT } \\
18(26.5 \%)\end{array}$} & \multirow{2}{*}{$\begin{array}{c}\text { VMAT } \\
50(73.5 \%)\end{array}$} \\
\hline & & & & \\
\hline Follow-up & Median [range] months & $64[1,154]$ & $110[4,154]$ & $55[1,129]$ \\
\hline \multirow{2}{*}{ Age } & Median [range] & $50[18,82]$ & $51[20,82]$ & $50[18,79]$ \\
\hline & Mean \pm SE & $53 \pm 2$ & $53 \pm 4$ & $52 \pm 2$ \\
\hline \multirow{2}{*}{ Gender } & Male & $57(83.8 \%)$ & $18(100.0 \%)$ & $39(78.0 \%)$ \\
\hline & Female & $11(16.2 \%)$ & $0(0.0 \%)$ & $11(22.0 \%)$ \\
\hline \multirow{3}{*}{ Performance status } & 0 & $54(79.4 \%)$ & $13(72.2 \%)$ & $41(82.0 \%)$ \\
\hline & 1 & $11(16.2 \%)$ & $3(16.7 \%)$ & $8(16.0 \%)$ \\
\hline & 2 & $3(4.4 \%)$ & $2(11.1 \%)$ & $1(2.0 \%)$ \\
\hline \multirow{3}{*}{ Histology } & Keratinizing SCC & $11(16.2 \%)$ & $2(11.1 \%)$ & $9(18.0 \%)$ \\
\hline & $\begin{array}{l}\text { Non-keratinizing differentiated } \\
\text { carcinoma }\end{array}$ & $10(14.7 \%)$ & $2(11.1 \%)$ & $8(16.0 \%)$ \\
\hline & $\begin{array}{l}\text { Non-keratinizing undifferentiated } \\
\text { carcinoma }\end{array}$ & $47(69.1 \%)$ & $14(77.8 \%)$ & $33(66.0 \%)$ \\
\hline \multirow{4}{*}{ T stage } & 1 & $16(23.5 \%)$ & $5(27.8 \%)$ & $11(22.0 \%)$ \\
\hline & 2 & $22(32.4 \%)$ & $8(44.4 \%)$ & $14(28.0 \%)$ \\
\hline & 3 & $9(13.2 \%)$ & $0(0.0 \%)$ & $9(18.0 \%)$ \\
\hline & 4 & $21(30.9 \%)$ & $5(27.8 \%)$ & $16(32.0 \%)$ \\
\hline \multirow{4}{*}{ N stage } & 0 & $8(11.8 \%)$ & $1(5.6 \%)$ & $7(14.0 \%)$ \\
\hline & 1 & $17(25.0 \%)$ & $3(16.7 \%)$ & $14(28.0 \%)$ \\
\hline & 2 & 35 (51.5\%) & $10(55.6 \%)$ & 25 (50.0\%) \\
\hline & 3 & $8(11.8 \%)$ & $4(22.2 \%)$ & $4(8.0 \%)$ \\
\hline \multirow{4}{*}{ TNM stage } & 1 & $2(2.9 \%)$ & $0(0.0 \%)$ & $2(4.0 \%)$ \\
\hline & 2 & $12(17.6 \%)$ & $3(16.7 \%)$ & $9(18.0 \%)$ \\
\hline & 3 & $29(42.6 \%)$ & $9(50.0 \%)$ & $20(40.0 \%)$ \\
\hline & $4 a$ & 25 (36.8\%) & $6(33.3 \%)$ & 19 (38.0\%) \\
\hline \multirow{2}{*}{ Induction CT } & No & $18(26.5 \%)$ & $5(27.8 \%)$ & $13(26.0 \%)$ \\
\hline & Yes & $50(73.5 \%)$ & $13(72.2 \%)$ & $37(74.0 \%)$ \\
\hline \multirow{2}{*}{ Concomitant CT } & No & $9(13.2 \%)$ & $3(16.7 \%)$ & $6(12.0 \%)$ \\
\hline & Yes & $59(86.8 \%)$ & 15 (83.3\%) & 44 (88.0\%) \\
\hline
\end{tabular}

3DCRT - 3-dimensional conformal radiotherapy; VMAT — volumetric modulated arc therapy; SE — standard error; SCC — squamous cell carcinoma; CT - chemotherapy

3DCRT patients were planned with multiple plans to deliver 50 Gy to the low-risk PTV and an additional boost of 20 Gy to the high-risk PTV, in fractions of $2 \mathrm{~Gy}$. The beam arrangement included only photon fields (mixed 6 and $18 \mathrm{MV}$ ) in a single isocentre setting, located at the matching plane of the upper and lower neck fields. In most of the cases the upper part of the volume was covered by lateral fields (shaped with and without exclusion of the spinal cord, in a sort of field-in-field setting), and the lower part by the anterior and posterior fields excluding the spinal cord. The field entrances were similar for the boost plan (in some cases only the lateral fields were used, depending on the tumour extension and nodal region involvement).
The patients treated with VMAT were planned for simultaneous integrated boost (SIB). Patients were treated in 33 fractions of 2.12 and 1.65 Gy to the high- and low-risk volumes, for total doses of $69.96 \mathrm{~Gy}$ and $54.45 \mathrm{~Gy}$, respectively, or in 30 fractions of 2.2 and 1.8 Gy to deliver 66 Gy and 54 Gy to the high- and low-risk PTVs, respectively. The VMAT plans were optimized with the Progressive Resolution Optimizer, PRO versions from 8.5 to 11. The arc arrangement was, in about two-thirds of the patients, of two arcs with collimator angles between $\pm 5^{\circ}$ and $\pm 35^{\circ}$, depending on the patient and target anatomy, or two arcs with collimator angle of $90^{\circ}$, overlapping in the longitudinal direction of at least $2 \mathrm{~cm}$. The field size was kept within $15 \mathrm{~cm}$ in the $\mathrm{X}$ direction to improve modulation. 
The remaining one-third of the plans had four arcs: two long ones with collimator angles between $\pm 5^{\circ}$ and $\pm 15^{\circ}$, and two with collimator angle of $90^{\circ}$, overlapping in the longitudinal direction of at least $2 \mathrm{~cm}$.

Dose planning goals for PTVs were: $95 \%$ of the prescribed dose should cover $95 \%$ of the PTV $\left(\mathrm{V}_{95 \%}>95 \%\right)$; near-to-maximum dose to high-risk PTV $\left(D_{2 \%}\right)$ below $105 \%$ of its prescription dose; near-to-maximum dose minimized for the low-risk PTV. The OARs goals were set according to institutional guidelines based on international suggestions. For OARs, near-to-maximum dose $\left(D_{0.1 \mathrm{~cm}^{3}}\right)$ to the spinal cord and brainstem below $45 \mathrm{~Gy}$ [17, $18]$ and 54 Gy $[19,20]$, respectively; regarding the eyes, the final goal was indeed $D_{\max }<10$ Gy to the lenses. Those OARs goals were pursued with all the techniques. The following additional objectives were included in the intensity modulation technique plans: mean dose to the parotids $<26 \mathrm{~Gy}$ to the full glands $[21,22]$, mean dose to the oral cavity $<30$ Gy [22], mean dose to the larynx $<43$ Gy $[23,24]$; the pharyngeal constrictor muscles and the submandibular glands were not included in the optimization process.

All the dose distributions were calculated with the Anisotropic Analytical Algorithm AAA (versions 8.5 to 11) on the planning CT (without contrast agent). The treatments used 6MV beams from either TrueBeam, Clinac DHX, or Unique linear accelerators (Varian Medical Systems, Palo Alto, USA) equipped with a Millennium 120-MLC.

The treatment included induction chemotherapy in 50 cases $(73.5 \%)$ and concomitant systemic treatment with cisplatin in 59 cases $(86.8 \%)$.

\section{Toxicity assessment and follow-up}

Acute toxicity, occurring during RT and in the following 3 months, and late toxicity reported after 3 months from the end of RT, were recorded and graded according to the Common Toxicity Criteria (CTCAE) version 4 [25].

During the treatment, all patients had a weekly clinical evaluation. Patient follow-up continued according to the schedule: one month after the treatment, then every 3 months for the first 3 years, every 6 months for years 4 and 5 and then yearly. At the first follow-up visit at least a post-treatment imaging was required. The treatment response was evaluated through CT, MRI and/or PET imaging as well as clinical assessment, and recorded according to RECIST criteria v. 1.2.

Toxicity data were compared with dosimetric parameters to assess possible correlations.

\section{Statistical analysis}

Standard descriptive statistics (mean standard deviation and cross-tabulation analysis) were used to describe the dosimetric and toxicity data.

The progression free (PFS) and overall survival (OS) were plotted by the Kaplan-Meier method, evaluating the time elapsed from the end of the radiotherapy to the last follow-up (censored data) or the specific event, and compared using the log-rank test. OS evaluated the deaths from any causes, while the PFS events were local, nodal or distant progression, or death from any causes. Multivariate Cox regression analysis included the variable with $\mathrm{p}$-values $\leq 0.05$ by univariate analysis. All data were analysed using the SPSS statistical software package (version 21, IBM Corporation, NY, USA).

\section{Results}

The median follow-up, calculated from the end of the RT treatment, was 64 months (range 3-154). At the time of the analysis, 52 patients of the cohort were alive. At the time of the last evaluation, complete remission was assessed in 46 cases (67.6\%). Locoregional relapse was recorded in 19 patients with a median time of 11 months (range 1-75 months). Among all locoregional relapses, 18 patients recurred inside the high dose region (high-risk PTV) and 2 patients in the low dose region (low-risk PTV). Distant metastases were developed in 6 patients with a median time of 7 months (range 2-45 months): 3 patients with liver lesions, 2 lung relapses, one bone metastases, and one distant lymph node metastases.

All patients, except one, completed the planned treatment; the median overall treatment time (OTT) was 51 days in the 35 fractions group (3DCRT), 49 and 43 days in the 33 and 30 fraction groups (VMAT), respectively.

The 5- and 10-year PFS rates were $62.7 \pm 6.5 \%$ and $53.2 \pm 8.7 \%$, respectively. The 5 - and 10 -year OS rates were $78.9 \pm 5.5 \%$ and $61.4 \pm 9.2 \%$, respectively.

At univariate analysis, performance status PS equal to $0(\mathrm{p}=0.02)$, lower TNM stage $(\mathrm{p}=0.03)$, 
and the use of concomitant chemotherapy $(\mathrm{p}=0.05)$ significantly improved PFS. Age $(\mathrm{p}=0.55)$, gender $(\mathrm{p}=0.85)$, histology $(\mathrm{p}=0.77)$, induction chemotherapy $(p=0.70)$, treatment technique $(p=0.25)$, dose fractionation $(\mathrm{p}=0.32)$ had no significant impact on PFS.

At the multivariate Cox analysis TNM stage $(p=0.02)$ and concomitant chemotherapy $(p=0.01)$ were significant for PFS.

Regarding OS, at the univariate analysis PS equal to $0(\mathrm{p}=0.01)$ and concomitant chemotherapy ( $\mathrm{p}=0.02)$ were significantly correlated. The technique of RT $(\mathrm{p}=0.38)$ and the dose fractionation $(\mathrm{p}=0.30)$ had no significant impact on OS. Visual OS (and PFS) trend for the patients treated with conventional fractionation of $2 \mathrm{~Gy} /$ fraction, or treated with hypofractionated SIB is presented in Figure 1. In the multivariate Cox analysis concomitant chemotherapy $(\mathrm{p}=0.04)$ remained significant.

Regarding acute toxicity, mucosal, salivary, deglutition and skin side effects are presented in Figure 2. A clear improvement with VMAT is observed, with moderate and severe acute toxici- ties ( $\geq$ G2) more common in the 3DCRT group. Acute $\geq$ G2 mucositis, xerostomia, dysphagia, and skin toxicities were $61 \%, 67 \%, 19 \%$, and $39 \%$ in the 3DCRT group, respectively, and $38 \%, 42 \%, 12 \%$, and $18 \%$ in the VMAT group, respectively.

The acute toxicity improvement with VMAT was supported by the ability of this technique to better spare OARs, as shown in Table 2. Odds ratio (OR) for acute salivary toxicity, between VMAT and 3DCRT, was $4.67(\mathrm{p}=0.02)$. Also, the other toxicities, as expected, presented OR in favour of VMAT, although not significant (but with a trend to be significant): mucositis OR of $2.56(\mathrm{p}=0.09)$, dysphagia OR of $2.76(\mathrm{p}=0.08)$, and skin toxicity OR of $2.90(\mathrm{p}=0.08)$.

The most important difference in the dose planned for the 3DCRT and VMAT groups concerned the parotid gland mean dose. In detail, it was $61.3 \pm 1.1 \mathrm{~Gy}$ and $28.4 \pm 0.8 \mathrm{~Gy}$ for the 3DCRT and VMAT group, respectively $(\mathrm{p}<0.001)$. Correcting each bin of the dose-volume histogram $(\mathrm{DVH})$ with the corresponding equivalent dose at 2 Gy (biological correction), and considering the

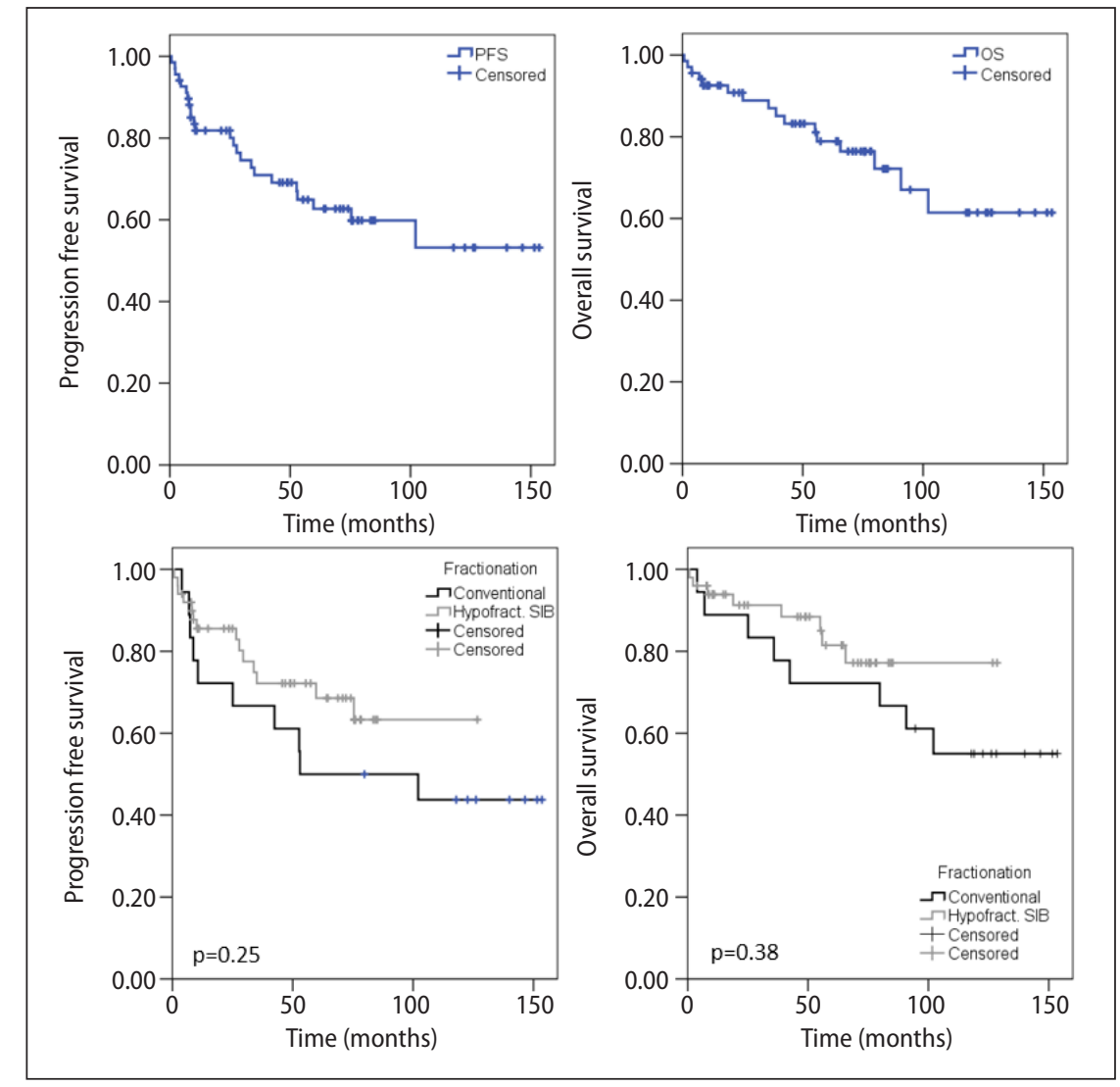

Figure 1. Kaplan-Meier curves for progression free survival and overall survival, for the whole population and according to radiotherapy fractionation [conventional and hypofractionated simultaneous integrated boost (SIB)] 


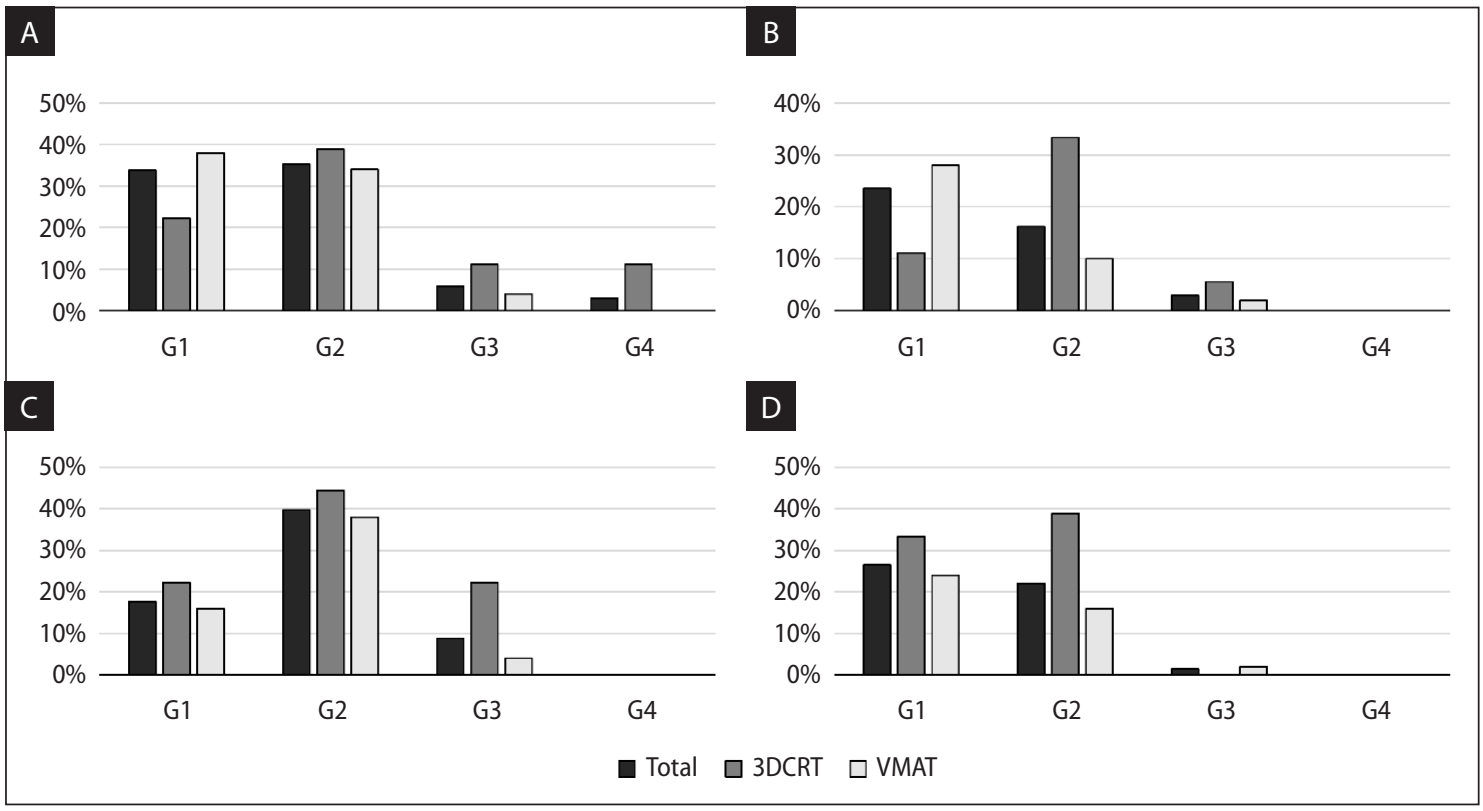

Figure 2. Acute toxicity, stratified for radiotherapy technique. A. Acute mucosal toxicity. B. Acute salivary toxicity. C. Acute dysphagia. D. Acute skin toxicity

Table 2. Dosimetric data

\begin{tabular}{|c|c|c|c|c|c|}
\hline Organ & Parameter & All patients & 3DCRT & VMAT & p-value \\
\hline \multirow[t]{2}{*}{ Parotid glands } & Mean Gy & $37.1 \pm 1.9$ & $61.3 \pm 1.1$ & $28.4 \pm 0.8$ & $<0.001$ \\
\hline & Mean Gy EQD2* & $32.5 \pm 2.1$ & $59.6 \pm 1.4$ & $22.7 \pm 0.8$ & $<0.001$ \\
\hline \multirow[t]{2}{*}{ Larynx } & Mean Gy & $40.1 \pm 1.0$ & $43.4 \pm 2.8$ & $38.9 \pm 0.9$ & 0.057 \\
\hline & Mean Gy EQD2* & $35.5 \pm 1.2$ & $41.7 \pm 3.0$ & $33.3 \pm 1.0$ & 0.001 \\
\hline \multirow[t]{2}{*}{ Oral cavity } & Mean Gy & $44.5 \pm 0.8$ & $48.0 \pm 1.4$ & $43.2 \pm 0.8$ & 0.005 \\
\hline & Mean Gy EQD2* & $40.6 \pm 0.9$ & $46.1 \pm 1.4$ & $38.6 \pm 1.0$ & $<0.001$ \\
\hline Submandibular glands & Mean Gy & $60.3 \pm 0.8$ & $62.6 \pm 0.9$ & $59.5 \pm 0.8$ & 0.021 \\
\hline
\end{tabular}

3DCRT - 3-dimensional conformal radiotherapy; VMAT — volumetric modulated arc therapy; EQD2 — equivalent 2 Gy fractions; *from biologically corrected dose-volume histograms (DVHs)

different fractionations with a/b ratio of $2 \mathrm{~Gy}$ (for each of the multiple plans in the 3DCRT cases), doses were $59.6 \pm 1.4 \mathrm{~Gy}$ and $22.7 \pm 0.8 \mathrm{~Gy}$ for the 3DCRT and VMAT group, respectively. In patients presenting G0 or G1 salivary toxicity, the mean parotid doses were $35.3 \pm 2.0 \mathrm{~Gy}(30.5 \pm 2.2$ Gy biologically corrected), compared with $44.6 \pm 4.6 \mathrm{~Gy}$ ( $40.9 \pm 5.3$ Gy biologically corrected) to the patients developing $\geq \mathrm{G} 2$ toxicity ( $\mathrm{p}=0.05$ ).

Correlations between dose parameters for OARs and acute toxicities ( $<\mathrm{G} 2 v$ s. $\geq \mathrm{G} 2)$ were evaluated with the ANOVA univariate analysis. Although the technique (3DCRT vs. VMAT) presents highly significant correlation with acute toxicities as well as the mean doses to the parotids, the larynx and the oral cavity, these significances do not directly trans- late to correlations between acute toxicities and mean OAR doses. Analysing the biologically corrected doses to account for the different fractionations, salivary toxicity correlates with the mean parotid dose $(\mathrm{p}=0.05)$, dysphagia with the mean laryngeal dose $(\mathrm{p}=0.04)$ and the mean oral cavity dose $(p=0.06)$, while no correlation was found for mucositis (all $\mathrm{p}>0.17$ ). In particular, the dysphagia OR ( $<$ G2 vs. $\geq$ G2) was 3.31 for the biologically corrected mean dose to the oral cavity $(\mathrm{a} / \mathrm{b}=3 \mathrm{~Gy})$ above $38 \mathrm{~Gy}(\mathrm{p}=0.024)$, and 3.30 for the biologically corrected mean dose to the larynx $(\mathrm{a} / \mathrm{b}=3 \mathrm{~Gy})$ above $33 \mathrm{~Gy}(\mathrm{p}=0.030)$.

In the late setting, none of the patients reported mucositis or skin toxicity. Late dysphagia and xerostomia are reported in Figure 3. Late dysphagia pre- 


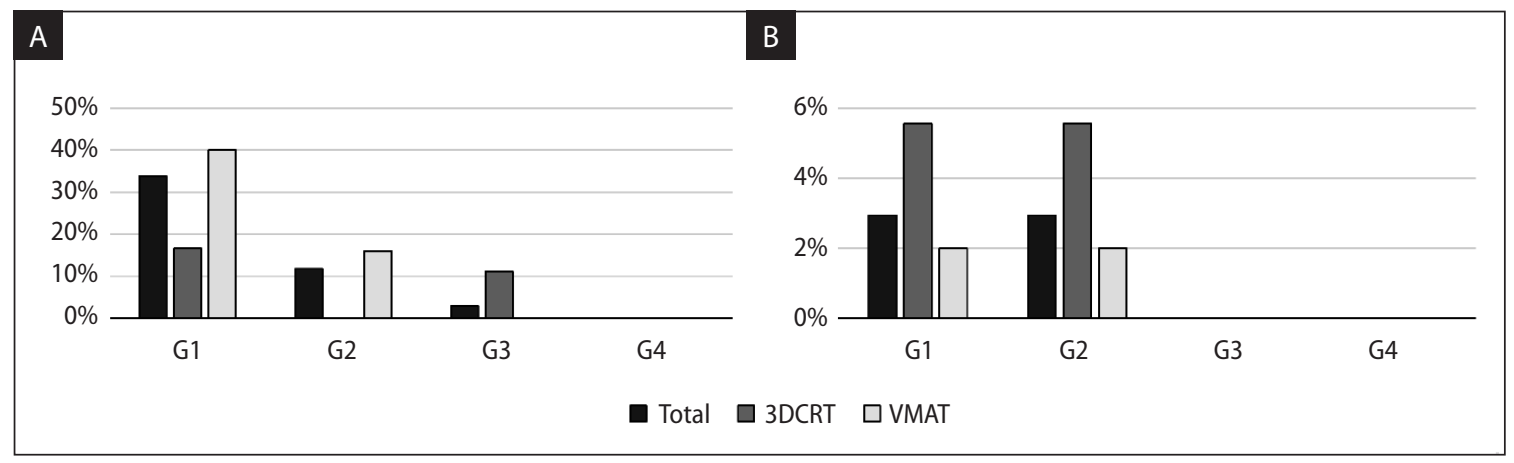

Figure 3. Late toxicity, stratified for radiotherapy technique. A. Late salivary toxicity. B. Late dysphagia

sented an OR of 3.00 in favour of VMAT, although with a p-value of 0.29 .

\section{Discussion}

In the last decade, the IMRT technique has become the standard approach for the irradiation of NPC. Alone or in combination with chemotherapy, IMRT is characterized by a better sparing of the healthy tissue with an improvement in target coverage compared to conventional 3DCRT and more obsolete 2DRT [26-28]. With the VMAT technique, the treatment time was reduced compared to standard IMRT keeping high sparing of OARs and target coverage.

Guo et al. [29] published in 2015 a series of 205 NPC cases treated with VMAT technique only. All patients were treated with a dose of 68-70 Gy in 30-33 fractions. With a median follow-up of 37.3 months, the 3 -year DFS and OS were $86.8 \%$ and $97.0 \%$, confirming the benefit of VMAT in this setting.

Here we reported outcomes in terms of disease control and toxicity of two groups of patients affected by NPC treated with the 3DCRT or VMAT techniques. At the best of our knowledge, this is the first study to compare these two approaches in the management of NPC.

The 5-year PFS and OS rates for the whole population were $62.7 \pm 6.5 \%$ and $78.9 \pm 5.5 \%$, respectively, with no statistical differences according to the RT technique. Our results are in accordance with the results of other studies reporting the traditional IMRT technique or 3DCRT [26, 30]. Kuang et al. [27] reviewed the treatment records of 182 patients treated with IMRT and 198 patients treated with 3DCRT. The 4-year disease-free survival (DFS) and
OS of the IMRT and 3DCRT groups were 74.7\% and $65.0 \%$, and 83.5 and $72.1 \%$, respectively. Also, Moretto et al. [28] compared patients treated with 2DRT/3DCRT with an IMRT group. A total of 52 patients were included showing 5-year OS and DFS rates of $79 \%$ and $65 \%$. No statistically significant difference was observed between the 2DRT/3DCRT and IMRT groups.

We previously compared two different hypofractionated schedules [31], demonstrating no differences in terms of PFS and OS, but a possible impact on dysphagia's incidence.

In our current study, a significant correlation with OS was observed for good PS $(\mathrm{p}=0.01)$ and concomitant chemotherapy $(\mathrm{p}=0.02)$, while the technique of RT resulted to have no impact ( $\mathrm{p}=0.38$ ). Concomitant chemotherapy was used in the majority of our cohort (86.8\%). A clear benefit from concomitant chemotherapy was demonstrated by previous meta-analyses in terms of survival improvement and lower risk of death [32-34], however, at the expense of an increased rate of side effects [35-37].

Therefore, the toxicity reduction seems to be relevant in this setting. In our study, a clear benefit in terms of toxicity was observed for the VMAT technique compared to the 3DCRT approach. Moderate and severe (> G2) acute skin toxicity, xerostomia, mucositis, and dysphagia were more common in the 3DCRT group compared to the VMAT group and this advantage is correlated with the ability of VMAT to better spare the main OARs. A significant dose reduction was observed for all the analysed healthy organs. As examples, we observed for parotid glands a physical mean dose reduction from 61.4 Gy to $28.4 \mathrm{~Gy}(\mathrm{p} \leq 0.001)$ and, for oral cavity, the volume receiving at least 60 Gy was reduced from $44.0 \%$ to $14.9 \%$ ( $\mathrm{p} \leq 0.001)$. 
Data published by other authors fail to correlate the OAR doses with the toxicity profile, and the doses delivered to the OARs are quite different in the various institutions and experiences. In the study by Moretto et al. [28], the average mean dose to the contralateral parotid (considered as the parotid gland opposite to main nasopharyngeal tumoral mass) was 32.8 Gy for the 3DCRT group and 31.8 Gy for the IMRT group. Pow et al. [38] reported dosimetric data for the only IMRT cohort for a small study comparing IMRT $v$ s. 3 DCRT treatment, as 42 Gy observed for the mean dose to the ipsilateral parotid gland, and 41.3 Gy for the contralateral one. In another study conducted by Wolden et al. [39], for 74 NPC patients treated with IMRT, precise dose calculation was available only for 42 patients, reporting an average mean dose to the parotid of $35.2 \mathrm{~Gy}$, and a reduction to $26 \mathrm{~Gy}$ in one third of the cohort without sacrificing target coverage.

In our cohort, although not large, very different doses to OARs were delivered to the patients, due to the different techniques. This variation, however, allowed us to try to determine possible correlations between the planned (and possibly delivered) dose and the toxicity. The different fractionation schemes used in the 3DCRT setting (treatment of the elective volume in 25 fractions and subsequent boost in 10 fractions) and with the VMAT technique (SIB in 33 or 30 fractions) makes it necessary to correct the dosimetric data according to the equivalent dose (at $2 \mathrm{~Gy} /$ fraction) to allow a reasonable comparison. Indeed, some differences become significant only in the case of biologically corrected doses, enforcing the need of the correction, and, on the other hand, enhancing the problem of the OAR dose fractionation and the shape of the specific DVH when dosimetric data of non-homogeneous irradiations has to be correlated with clinical results. Related to such a correction is the $\mathrm{a} / \mathrm{b}$ value of the specific organ, which suffers from great uncertainty. The lower the ratio, the larger the difference between the physical and biologically corrected dose for inhomogeneous dose distribution, as is the case of intensity-modulated treatments. This issue should not be neglected, but the current planning systems report physical doses, and those values are today the basis for plan evaluation.

Once having clarified the problem, and considering the below listed limitations of the current study, our work allowed suggesting dosimetric parameters as possible predictors for toxicities.

Moreover, it is clear that the single toxicities cannot be related to the irradiation of a single organ. The physiological interactions between organs is a known process, and the data here presented suggests, for example, that it is not sufficient to reduce the mean dose to the parotids below the level of 26 or $20 \mathrm{~Gy}$, as suggested by the guidelines, to lower the probability of causing low-grade xerostomia. Also, the dose to other structures, for example the oral cavity, will contribute in a complex way to toxicity that is commonly assigned to the parotid irradiation [40].

Some issues, however, limit the present study, including the retrospective nature of the analysis, the small sample size, and the heterogeneity among the two groups of treatment.

\section{Conclusion}

The present study confirms the significant benefit of the VMAT technique compared with 3DCRT in terms of side effect reduction for the treatment of NPC. Disease control and survival rates were not affected by this modern RT approach. Considering that 3DCRT is still in use in developing and several developed countries, we think that this information could be relevant to reinforce the concept of usefulness of modern RT techniques for such complex anatomical areas. Finally, correlations were found between salivary toxicity and mean parotid dose, and between dysphagia and mean biologically corrected doses to the larynx and oral cavity.

\section{Conflicts of interest/competing interests}

L.C. acts as a Scientific Advisor to Varian Medical Systems and is Clinical Research Scientist at $\mathrm{Hu}-$ manitas Research Hospital. The other authors have no conflicts of interest to disclose.

\section{Funding}

This research did not receive any specific grant from funding agencies in the public, commercial, or not-for-profit sectors.

\section{Ethics approval}

All patients were treated in agreement with the Helsinki declaration. Informed consent was obtained 
from all individual participants included in this study.

\section{Availability of data and material}

The whole material is available upon request.

\section{References}

1. Parkin DM, Whelan SL, Ferlay J, et al. International Agency for Cancer Research. Cancer incidence in five continents, Vol. VIII. IARC Sci Publ. 2009; 155: 1-781, indexed in Pubmed: 12812229.

2. Chua $M$, Wee J, Hui E, et al. Nasopharyngeal carcinoma. Lancet. 2016; 387(10022): 1012-1024, doi: 10.1016/s01406736(15)00055-0, indexed in Pubmed: 26321262.

3. Setton J, Han J, Kannarunimit D, et al. Long-term patterns of relapse and survival following definitive intensity-modulated radiotherapy for non-endemic nasopharyngeal carcinoma. Oral Oncol. 2016; 53: 67-73, doi: 10.1016/j. oraloncology.2015.11.015, indexed in Pubmed: 26675283.

4. Stenmark MH, McHugh JB, Schipper M, et al. Nonendemic HPV-positive nasopharyngeal carcinoma: association with poor prognosis. Int J Radiat Oncol Biol Phys. 2014; 88(3): 580-588, doi: 10.1016/j.ijrobp.2013.11.246, indexed in Pubmed: 24521676.

5. Huang K, Xia P, Chuang C, et al. Intensity-modulated chemoradiation for treatment of stage III and IV oropharyngeal carcinoma: the University of California-San Francisco experience. Cancer. 2008; 113(3): 497-507, doi: 10.1002/ cncr.23578, indexed in Pubmed: 18521908.

6. Miah AB, Bhide SA, Del Rosario L, et al. Induction Chemotherapy Followed by Chemo-intensity-modulated Radiotherapy for Locally Advanced Nasopharyngeal Cancer. Clin Oncol (R Coll Radiol). 2016; 28(8): e61-e67, doi: 10.1016/j. clon.2016.01.012, indexed in Pubmed: 26876458.

7. Belgioia L, Bacigalupo A, Vecchio $S$, et al. Excellent survival regardless of disease stage in patients with advanced nasopharyngeal cancer. Tumori. 2016; 102(4): 381-386, doi: 10.5301/tj.5000483, indexed in Pubmed: 27056334.

8. Kam MKM, Leung SF, Zee B, et al. Prospective randomized study of intensity-modulated radiotherapy on salivary gland function in early-stage nasopharyngeal carcinoma patients.J Clin Oncol. 2007; 25(31):4873-4879, doi: 10.1200/ JCO.2007.11.5501, indexed in Pubmed: 17971582.

9. MCMillan AS, Pow EHN, Kwong DLW, et al. Preservation of quality of life after intensity-modulated radiotherapy for early-stage nasopharyngeal carcinoma: results of a prospective longitudinal study. Head Neck. 2006; 28(8): 712-722, doi: 10.1002/hed.20378, indexed in Pubmed: 16475203.

10. Palma DA, Verbakel WF, Otto $K$, et al. New developments in arc radiation therapy: a review. Cancer Treat Rev. 2010; 36(5): 393-399, doi: 10.1016/j.ctrv.2010.01.004, indexed in Pubmed: 20181430.

11. Yu C, LiX, Ma L, et al. Clinical implementation of intensitymodulated arc therapy. Int J Radiat Oncol Biol Phys. 2002; 53(2): 453-463, doi: 10.1016/s0360-3016(02)02777-3, indexed in Pubmed: 12023150.

12. Fenwick JD, Tomé WA, Soisson ET, et al. Tomotherapy and other innovative IMRT delivery systems. Semin
Radiat Oncol. 2006; 16(4): 199-208, doi: 10.1016/j.semradonc.2006.04.002, indexed in Pubmed: 17010902.

13. Leech M, Coffey M, Mast M, et al. ESTRO ACROP guidelines for positioning, immobilisation and position verification of head and neck patients for radiation therapists. Tech Innov Patient Support Radiat Oncol. 2017; 1: 1-7, doi: 10.1016/j. tipsro.2016.12.001, indexed in Pubmed: 32095536.

14. ESTRO ACRP. Guidelines for positioning, immobilisatio and position verification of head and neck patients for RTTS. https://www.estro.org/ESTRO/media/ESTRO/ About/Committees/RTT/guidelines-for-positioningimmobilisation-and-position-verification-of-head-andneck-patients-for-rtts.pdf.

15. Grégoire V, Levendag P, Ang KK, et al. CT-based delineation of lymph node levels and related CTVs in the nodenegative neck: DAHANCA, EORTC, GORTEC, NCIC,RTOG consensus guidelines. Radiother Oncol. 2003; 69(3): 227-236, doi: 10.1016/j.radonc.2003.09.011, indexed in Pubmed: 14644481.

16. Grégoire V, Ang K, Budach W, et al. Delineation of the neck node levels for head and neck tumors: a 2013 update. DAHANCA, EORTC, HKNPCSG, NCIC CTG, NCRI, RTOG, TROG consensus guidelines. Radiother Oncol. 2014; 110(1): 172-181, doi: 10.1016/j.radonc.2013.10.010, indexed in Pubmed: 24183870.

17. Kirkpatrick JP, van der Kogel AJ, Schultheiss TE. Radiation dose-volume effects in the spinal cord. Int J Radiat Oncol Biol Phys. 2010; 76(3 Suppl): S42-S49, doi: 10.1016/j. ijrobp.2009.04.095, indexed in Pubmed: 20171517.

18. Marcus R, Million R. The incidence of myelitis after irradiation of the cervical spinal cord. Int J Radiat Oncol Biol Phys. 1990; 19(1): 3-8, doi: 10.1016/0360-3016(90)90126-5, indexed in Pubmed: 2380091.

19. Mayo C, Yorke E, Merchant TE. Radiation associated brainstem injury. Int J Radiat Oncol Biol Phys. 2010; 76(3 Suppl): S36-S41, doi: 10.1016/j.ijrobp.2009.08.078, indexed in Pubmed: 20171516.

20. Hoppe BS, Stegman LD, Zelefsky MJ, et al. Treatment of nasal cavity and paranasal sinus cancer with modern radiotherapy techniques in the postoperative setting--the MSKCC experience. Int J Radiat Oncol Biol Phys. 2007; 67(3):691-702, doi: 10.1016/j.jirobp.2006.09.023, indexed in Pubmed: 17161557.

21. Deasy JO, Moiseenko V, Marks L, et al. Radiotherapy dosevolume effects on salivary gland function. Int J Radiat Oncol Biol Phys. 2010; 76(3 Suppl): S58-S63, doi: 10.1016/j. ijrobp.2009.06.090, indexed in Pubmed: 20171519.

22. Eisbruch A, Kim HM, Terrell JE, et al. Xerostomia and its predictors following parotid-sparing irradiation of headand-neck cancer. Int J Radiat Oncol Biol Phys. 2001; 50(3): 695-704, doi: 10.1016/s0360-3016(01)01512-7, indexed in Pubmed: 11395238.

23. Rancati T, Schwarz M, Allen AM, et al. Radiation dosevolume effects in the larynx and pharynx. Int J Radiat Oncol Biol Phys. 2010; 76(3 Suppl): S64-S69, doi: 10.1016/j. ijrobp.2009.03.079, indexed in Pubmed: 20171520.

24. Sanguineti G, Adapala P, Endres EJ, et al. Dosimetric predictors of laryngeal edema. Int J Radiat Oncol Biol Phys. 2007; 68(3): 741-749, doi: 10.1016/j.ijrobp.2007.01.010, indexed in Pubmed: 17398024.

25. Common Terminology Criteria for Adverse Events (CTCAE). Version 4.0. (v4.03: June 14, 2010). http://evs.. 
nci.nih.gov/ftp1/CTCAE/CTCAE_4.03_2010-06-14_ QuickReference_8.5x11.pdf.

26. Fang FM, Chien CY, Tsai WL, et al. Quality of life and survival outcome for patients with nasopharyngeal carcinoma receiving three-dimensional conformal radiotherapy vs. intensity-modulated radiotherapy-a longitudinal study. Int J Radiat Oncol Biol Phys. 2008; 72(2): 356-364, doi: 10.1016/j.ijrobp.2007.12.054, indexed in Pubmed: 18355980.

27. Kuang WLu, Zhou Q, Shen LF. Outcomes and prognostic factors of conformal radiotherapy versus intensity-modulated radiotherapy for nasopharyngeal carcinoma. Clin TransI Oncol. 2012; 14(10): 783-790, doi: 10.1007/s12094012-0864-5, indexed in Pubmed: 22855156.

28. Moretto F, Rampino M, Munoz F, et al. Conventional 2D (2DRT) and 3D conformal radiotherapy (3DCRT) versus intensity-modulated radiotherapy (IMRT) for nasopharyngeal cancer treatment. Radiol Med. 2014; 119(8): 634-641, doi: 10.1007/s11547-013-0359-7, indexed in Pubmed: 24424659.

29. Guo R, Tang LL, Mao YP, et al. Clinical Outcomes of Volume-Modulated Arc Therapy in 205 Patients with Nasopharyngeal Carcinoma: An Analysis of Survival and Treatment Toxicities. PLoS One. 2015; 10(7): e0129679, doi: 10.1371/journal.pone.0129679, indexed in Pubmed: 26146828.

30. Spiotto MT, Weichselbaum RR. Comparison of 3D confromal radiotherapy and intensity modulated radiotherapy with or without simultaneous integrated boost during concurrent chemoradiation for locally advanced head and neck cancers. PLoS One. 2014; 9(4): e94456, doi: 10.1371/ journal.pone.0094456, indexed in Pubmed: 24714187.

31. Franzese C, Fogliata A, Franceschini D, et al. Impact of hypofractionated schemes in radiotherapy for locally advanced head and neck cancer patients. Laryngoscope. 2020; 130(4): E163-E170, doi: 10.1002/lary.28048, indexed in Pubmed: 31063588.

32. Baujat B, Audry H, Bourhis J, et al. MAC-NPC Collaborative Group. Chemotherapy in locally advanced nasopharyngeal carcinoma: an individual patient data meta-analysis of eight randomized trials and 1753 patients. Int J Radiat Oncol Biol Phys. 2006; 64(1): 47-56, doi: 10.1016/j. ijrobp.2005.06.037, indexed in Pubmed: 16377415.

33. Langendijk JA, Leemans CR, Buter J, et al. The additional value of chemotherapy to radiotherapy in locally ad- vanced nasopharyngeal carcinoma: a meta-analysis of the published literature. J Clin Oncol. 2004; 22(22): 4604-4612, doi: 10.1200/JCO.2004.10.074, indexed in Pubmed: 15542811.

34. Huncharek M, Kupelnick B. Combined Chemoradiation Versus Radiation Therapy Alone in Locally Advanced Nasopharyngeal Carcinoma. Am J Clin Oncol. 2002; 25(3): 219-223, doi: 10.1097/00000421-200206000-00002, indexed in Pubmed: 12040275.

35. Xie FY, Qi SN, Hu WH, et al. [Comparison of efficacy of docetaxel combined cisplatin (TP regimen) and cisplatin combined 5-fluorouracil (PF regimen) on locally advanced nasopharyngeal carcinoma]. Ai Zheng. 2007; 26(8): 880-884, indexed in Pubmed: 17697552.

36. Huang PY, Mai HQ, Luo DH, et al. Induction-concurrent chemoradiotherapy versus induction chemotherapy and radiotherapy for locoregionally advanced nasopharyngeal carcinoma. Ai Zheng. 2009; 28(10): 1033-1042, doi: 10.5732/cjc.009.10114, indexed in Pubmed: 19799810.

37. Hui EP, Ma BB, Leung SF, et al. Randomized phase II trial of concurrent cisplatin-radiotherapy with or without neoadjuvant docetaxel and cisplatin in advanced nasopharyngeal carcinoma. J Clin Oncol. 2009; 27(2): 242-249, doi: 10.1200/JCO.2008.18.1545, indexed in Pubmed: 19064973.

38. Pow EHN, Kwong DLW, McMillan AS, et al. Xerostomia and quality of life after intensity-modulated radiotherapy vs. conventional radiotherapy for early-stage nasopharyngeal carcinoma: initial report on a randomized controlled clinical trial. Int J Radiat Oncol Biol Phys. 2006; 66(4): 981-991, doi: 10.1016/j.ijrobp.2006.06.013, indexed in Pubmed: 17145528.

39. Wolden SL, Chen WC, Pfister DG, et al. Intensitymodulated radiation therapy (IMRT) for nasopharynx cancer: update of the Memorial Sloan-Kettering experience. Int J Radiat Oncol Biol Phys. 2006; 64(1): 57-62, doi: 10.1016/j.ijrobp.2005.03.057, indexed in Pubmed: 15936155.

40. Orlandi E, lacovelli NA, Rancati T, et al. Multivariable model for predicting acute oral mucositis during combined IMRT and chemotherapy for locally advanced nasopharyngeal cancer patients. Oral Oncol. 2018; 86: 266-272, doi: 10.1016/j.oraloncology.2018.10.006, indexed in Pubmed: 30409311. 